\title{
Neural network principle of implementation of digital filters
}

\author{
Sergey Morozov ${ }^{1}$, Gennady Makarov ${ }^{2}$ and Konstantin Kuzmin ${ }^{1, *}$ \\ ${ }^{1}$ Moscow state University of technology and management. K. G. Razumovsky (Smolensk branch), \\ Lenin street 77, 215100, Smolensk region, Vyazma, Russia \\ ${ }^{2}$ Smolensk Humanitarian University, Herzen street 2, 214023, Smolensk, Russia
}

\begin{abstract}
Comparative evaluations of the frequency responses (FR) of two types of filters implemented by the classical and neural network methods are carried out. It is shown that the neural network principle of the implementation of digital filters can serve as an alternative to the classical method for specifically defined parameters of FR in the pass bands and attenuation bands of the frequencies of signal spectrum. The simplest method for calculating the parameters of the filters' difference equations is the neural network approach, regardless of the type of classification of discrete and digital filters. The implementation of TM (transmultiplexer) on a digital element base requires the use of methods of filtering, modulating and demodulating signals that are largely different from traditional analog methods. The frequency responses of non-recursive types of filters presented in the paper are based on the property of the approximable function determined only in the pass bands and attenuation bands of the frequencies of signal spectrum.
\end{abstract}

\section{Introduction}

Since the 1970s, the ideas and methods of digital signal processing in radio engineering and telecommunications are receiving increasing attention. Significant progress has been made in the development of transmultiplexers (TM) - digital devices interfacing communication systems with time and frequency division of channels.

Implementation of TM on a digital element base requires the use of digital methods of filtering, modulating and demodulating signals that are largely different from traditional analog methods [1].

The basic methods of analyzing and synthesizing digital filters (DF) are described in a number of published papers [2], including the work where the DF is mathematically described by a difference equation (finite difference equation). The tasks of realizing the DF were reduced to the calculation of coefficients of difference equations for non-recursive and recursive filters. Its solution is usually performed by various classical methods, namely: 1) firstly, the filter type is defined, and then its transfer function is found; 2) firstly, the transfer function is set, which provides the necessary filtering properties in the frequency range, and then the filter is restored from it.

*Corresponding author: vskanhva@mail.ru 


\section{Materials and Methods}

\subsection{Statement of the problem}

The following approaches are considered in the article: 1. Normalized boundary frequencies of the filter's pass band - W p.b. and attenuation band - W a.b. are given. 2 . Calculate the coefficients of the transfer function in the form of some approximating function by analytical (classical) and neural network methods. 3. By modeling the filtering process, compare the frequency responses (FR) for two types of DF in the pass band and attenuation band of the frequency signal.

\subsection{Solution of the problem}

To solve the problem, it is necessary to construct a continuous approximable function that extends definition of the following function

$$
\mathrm{B}(\mathrm{W})=\left\{\begin{array}{cl}
1 & \text { when } 0<\mathrm{W}<\mathrm{W} \text { p.b. } \\
\frac{W p \cdot \text { Wa.b. }-W a \cdot b .}{\text { Wp. }} & \text { when } \mathrm{W} \text { p.b. }<\mathrm{W}<=\mathrm{W} \text { a.b. } \\
0 & \text { when } \mathrm{W} \text { a.b. }<=\mathrm{W}<=0.5,
\end{array}\right.
$$

where $\mathrm{W}$ - normalized frequency of input signal $\mathrm{f}$.

There are many different ways to extend definition of the function (1). We consider the simplest method in which the approximable function in the intermediate band linearly depends on $\mathrm{W}$. It is required to determine the values of the parameters $\mathrm{C}_{0}, \mathrm{C}_{1}, \mathrm{C}_{2}, \ldots \ldots, \mathrm{Ck}$ of the continuous approximating function $\mathrm{F}\left(\mathrm{W}, \mathrm{C}_{0}, \mathrm{C}_{1}, \mathrm{C}_{2}, \ldots, \mathrm{Ck}\right)$, so that for values of $\mathrm{W}$ the approximate equality holds

$$
\mathrm{F}\left(\mathrm{W}, \mathrm{C}_{0}, \mathrm{C}_{1}, \mathrm{C}_{2}, \ldots \ldots, \mathrm{Ck}\right) \approx \mathrm{B}(\mathrm{W}) .
$$

The independent dimensionless variable $\mathrm{W}$ is represented in the form

$$
\mathrm{W}=\frac{\omega T}{2 \pi},
$$

where $\omega=2 \pi f, \mathrm{~T}$ - signal sampling period. We agree that the variable $\mathrm{W}$, on demand of the reproducibility of the lattice function of the signal, takes values on the interval $[0 \div 0.5]$, which we call an interval of approximation.

Let us consider a non-recursive filter with an exactly linear phase response (PR). In this case, the approximating function in (2) can have the form of a trigonometric polynomial [3]

$$
\mathrm{F}(\mathrm{W}, \mathrm{C})=\sum_{k=0}^{n} C_{k} \operatorname{COS}(k 2 \pi W)
$$

It is assumed that the coefficients $\mathrm{Ci}=\left(\mathrm{C}_{0}, \mathrm{C}_{1}, \mathrm{C}_{2}, \ldots, \mathrm{Ck}\right)$ of the function $\mathrm{F}(\mathrm{W}, \mathrm{C})$ coincide with the parameters of the filter or are related to them by a fairly simple dependence. Assume that the parameters $\mathrm{C}_{0}, \mathrm{C}_{1}, \mathrm{C}_{2}, \ldots \ldots ., \mathrm{Ck}$, of the function $\mathrm{F}(\mathrm{W}, \mathrm{C})$ coincide or are simply related to the parameters of the filter.

Then the equation of a non-recursive filter in finite differences will have the form 


$$
\mathrm{Yi}[\mathrm{nT}]=\sum_{k=0}^{L=14} C_{k} X[n T-k T], \quad \mathrm{i}=1,2
$$

where $\mathrm{X}[\mathrm{nT}]$ and $\mathrm{Y}[\mathrm{nT}]-\mathrm{n}$-e are references of the input and output signals of the filter, respectively, which determine the operation of the digital filter in reality. Calculation of the coefficient vector $\mathrm{Ci}$ is performed by classical methods on the basis of the expansion of the approximable function ( 1 ) into a Fourier series $(i=1)$ and representing a Fourier series by the neural network $(i=2)$.

The parameters of the neural network are calculated using the linear function of the neuron activation according to the algorithm:

$$
\mathrm{C}_{\mathrm{k}}[\mathrm{n}]=\mathrm{C}_{\mathrm{k}}\left[\mathrm{n} \_1\right]+\mathrm{hgkCOS}(\mathrm{k} 2 \pi \mathrm{XF}), \quad(\mathrm{k}=0, \mathrm{n})
$$

where $\mathrm{XF}=\mathrm{fT}, \mathrm{gk}=\mathrm{B}(\mathrm{W})-\mathrm{F}(\mathrm{C}, \mathrm{W}), \quad \mathrm{h}$ - the parameter of the learning speed of the neural network, which takes values less than one. The frequency range of the input signal is $0<=\mathrm{W}<=\mathrm{Wmax}$, the signal sampling rate is $\mathrm{f}_{\mathrm{d}}=\frac{1}{T}$.

\section{Results}

\subsection{Non-recursive options of digital filters}

The frequency response of the filter is expressed through the ratio of the signal power at the filter output to the signal power at the filter input.

Of interest is the frequency responses (A (w)) of a non-recursive filter with model (4) and its deviation from unity in the pass band and from zero in the attenuation band. The results of modeling the filtering process with a low-pass filter for the variants when $i=1$ (frequency response curve of the classical filter) and $i=2$ (frequency response curve of the neural network filter) are shown in Fig.1.

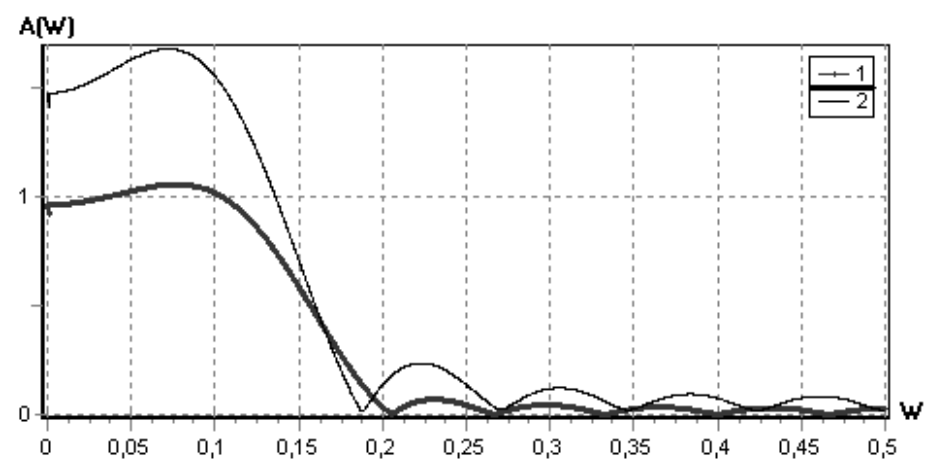

Fig. 1. Dependencies of the frequency responses for two low-pass filters on the normalized frequency $\mathrm{W}$ when Wp.b. $=0.125$ and $\mathrm{W}$ a.b. $=0.1875$.

It follows from Figure1.1. that the frequency responses in the pass band are not flat.

If the neural network filter coefficients are calculated when learning a neural network in the mode of a recursive scheme, and the filter is implemented as a non-recursive one, then, as a result of decreasing the values of parameters of the function $\mathrm{F}(\mathrm{W}, \mathrm{C})$, the frequency response in the pass band is usually reduced [4]. 
With the extension of the frequency range in the pass band, the dependencies of the frequency responses for two low-pass filters are illustrated in Fig. 2.

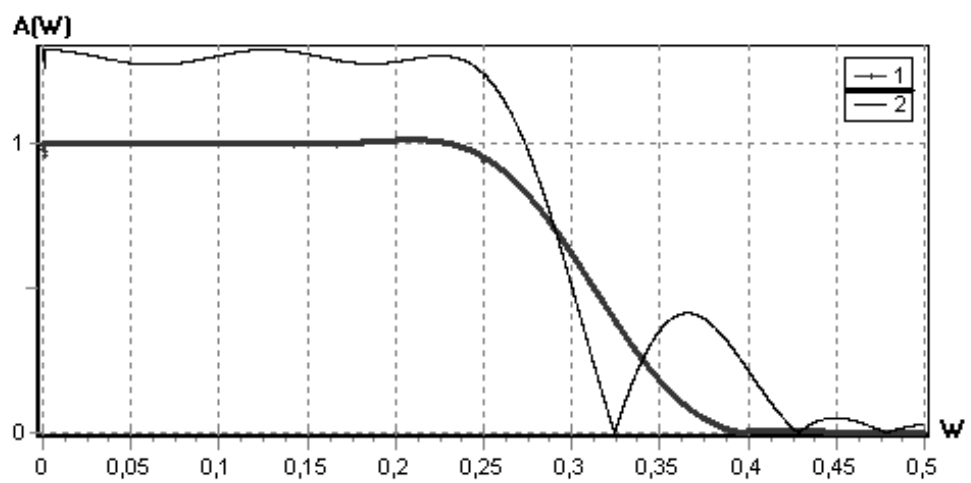

Fig. 2. Dependencies of the frequency response for two low-pass filters on the normalized frequency $\mathrm{W}$ when Wp.b. $=0.25$ and $\mathrm{W}$ a.b. $=0.375$.

The implementation of band pass filters according to the classical (curve 1) and neural network (curve 2) schemes is illustrated in Fig. 3. The non-recursive neural network filter is described by the difference equation (4), the coefficients of which are obtained when learning a neural network in the non-recursion mode.

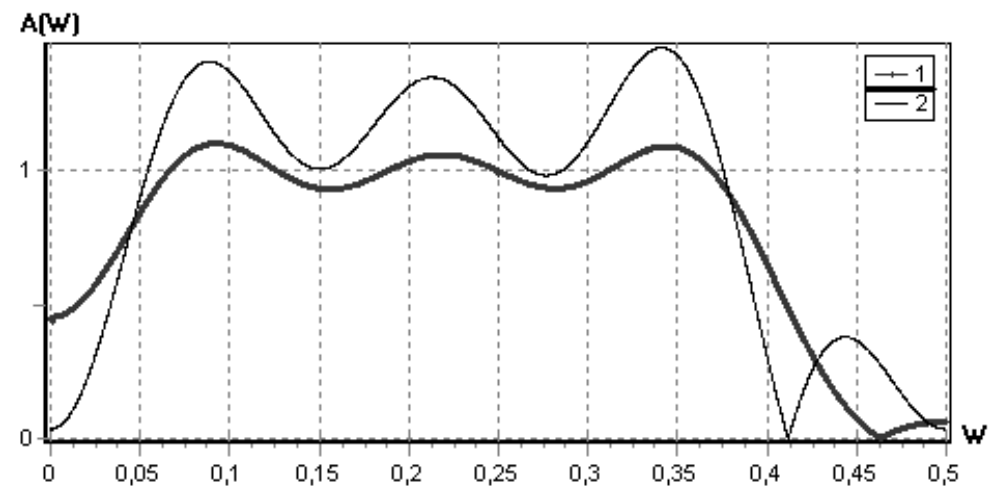

Fig. 3. Dependencies of the frequency responses for two low-pass band filters on the normalized frequency $\mathrm{W}$ when Wp.b.1.=0.0375, Wp.b. $2=0.3875$ and $\mathrm{W}$ a.b. $=0.4375$.

Thus, the neural network non-recursive filter with respect to the classical non-recursive filter has an excessive frequency response (FR) and shows more precise fixation of the specified boundary values when choosing this type of filter.

On the other hand, with the expansion of the number of summands in (4) in the limit of, as a rule, no more than $\mathrm{L}<=15$, the dependencies of the frequency responses for the classical and neural network filter types slightly differ from each other. In particular, for the learning scheme with $\mathrm{L}=30$, only half of the summands were used in the difference equation (4). In this case, the frequency response for the two types of filters is illustrated in Fig. 4. 


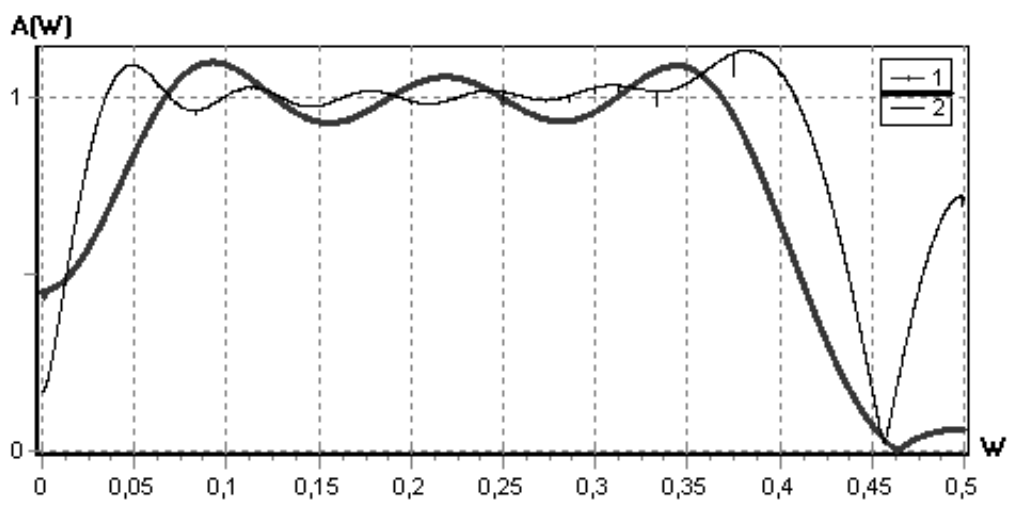

Fig. 4. Dependencies of the frequency responses for two low-pass band filters on the normalized frequency $\mathrm{W}$ when Wp.b1. $=0.0375$, Wp.b2 $=0.3875$ and $\mathrm{W}$ a.b. $=0.4375$.

The presented frequency responses of non-recursive filter types were based on the property of the approximable function (1) defined only in the pass bands and the attenuation bands of the frequencies of signal spectrum.

Consider an approximable function for a filter with several pass bands. To do this, we supplement (1) with the following conditions

$$
\mathrm{B}(\mathrm{W})=\left\{\begin{array}{cc}
1 & \text { when } 0<\mathrm{W}<\mathrm{Wp} . \mathrm{b} 0 \\
\frac{W-W_{A . B 0}}{W_{P . B 0}-W_{A . B 0}} & \text { when Wp.b.0 }<\mathrm{W}<\text { Wa.b0 }
\end{array}\right.
$$

Figure 5 illustrates the frequency responses of two non-recursive band-stop filters with two pass bands and one attenuation band within the boundaries of Wa.b0 and Wa.b1.

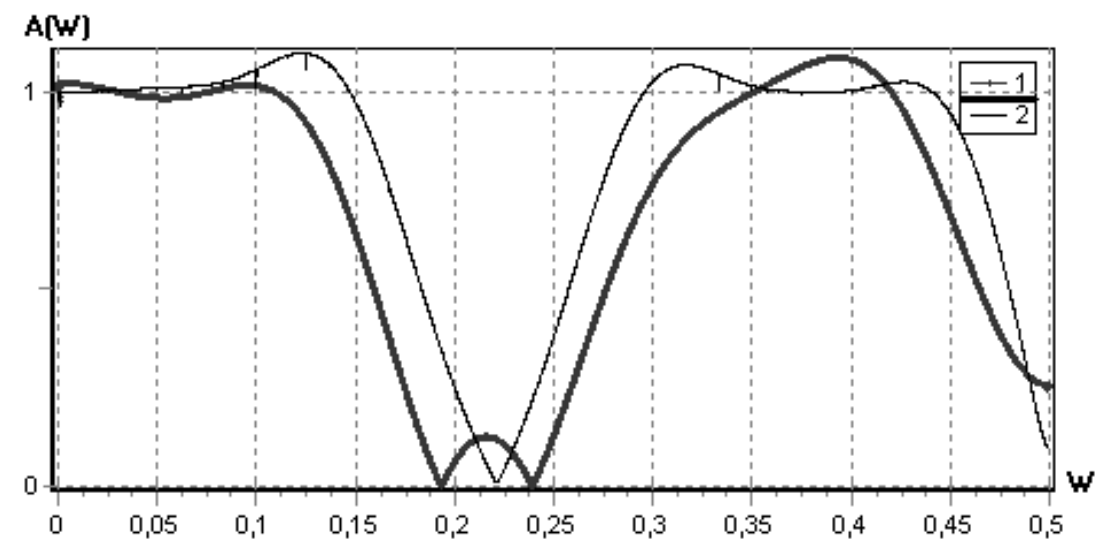

Fig. 5. Dependencies of the frequency responses for two band-stop filters from the normalized frequency $\mathrm{W}$ when Wp.b0 $=0.125$, Wa.b0 $=0.1875$, Wa.b1 $=0.25$ Wp.b1 $=0.3125$, Wp.b2 $=0.4375$ and Wa.b2 $=0.5$.

The Neural network band-stop filter in Fig. 5 (curve 2) has a wider pass band in comparison with the classical filter (curve 1) because of the width reduction of the attenuation band. 


\subsection{Recursive filter}

It is known that the classical method of analysis and synthesis of the DF is based mainly on a non-recursive type of filter [5]. Synthesis using a recursive filter is usually performed by preliminary finding the transfer function of a stable analog prototype filter [6]. For this purpose, as a rule, one or another guide to analog filters is used [7, 8].

The recursive filter, unlike the classical approach to approximation of the function (1), is easy to implement if the approximate equation (2) is performed on the basis of the neural network principle according to the algorithm (5) with preliminary learning of the neural network.

Let us consider an example with a recursive filter implemented using the neural network principle, and the classical variant using the expansion of the approximated function (1) in a Fourier series. The results are estimated for a band-stop filter with one pass band, which has the following filter parameters: Wp.b1 $=0.0375$, Wp.b2 $=0.3375$ and Wa.b2 $=0.425$.

The result of modeling of the filtering process is estimated by the recursive model

$$
\mathrm{Y}_{2}[\mathrm{nT}]=\sum_{k=0}^{L=15} C_{k} X[n T-k T]+\sum_{j=1}^{2} b_{j} Y_{2}[n T-j T],
$$

And the result is presented in Fig. 6.

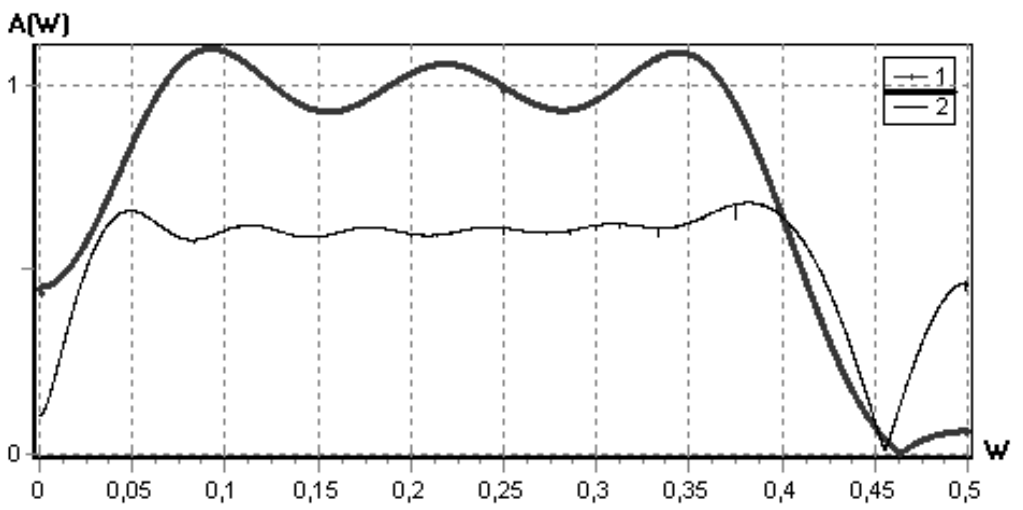

Fig. 6. Dependencies of the frequency responses for two low-pass band filters on the normalized frequency $\mathrm{W}$ when Wp.b1. $=0.0375$, Wp.b2 $=0.3875$ and $\mathrm{W}$ a.b. $=0.4375$.

\section{Discussion}

Summarizing the given data on the formation of the frequency response of the two types of filters, it should be noted that the recursive filter always has a greater attenuation of the signal in the pass band than the non-recursive filter. The recursion procedure, as a rule, equalizes the frequency response in the pass band of the signal. With the increase of the recursion summands, the uniformity of the frequency response in the pass band is violated. Therefore, it is more rational to consider the approach when the recursion procedure is implemented using the neural network scheme for calculating the parameters (6), and the filter implementation is performed according to the scheme (4). An example of such an approach is shown in Fig. 6.

Analysis of the properties of the digital filter realized by the neural network scheme showed that its phase response (PR) has a linear dependence. 
Analysis of two methods for implementation of digital recursive and non-recursive filters shows that the neural network principle, in comparison with the classical method, does not contain difficulties in determining the filter transfer function with the specified filtering requirements for the signal. Comparing the accuracy properties of non-recursive and recursive filters, we note that the recursive filter in the pass band has a greater uniformity of the frequency response. 10]

The stability of processes in a recursive digital filter is determined by the condition [9,

$$
\max |q(n T)|<1
$$

where $\mathrm{q}(\mathrm{nT})=\frac{-v^{m+2} \sin (n+1) \varphi}{\sin \varphi} ; v=\sqrt{b_{2}} ; \varphi=\arccos \left(-\frac{b_{1}}{2 v}\right) ; \mathrm{n}=1,2$.

The parameters of pole of the transfer function (6) for the low-pass filter are, respectively, $\mathrm{b}_{1}=0.11482$ and $\mathrm{b}_{2}=0.09448$. Thus, condition (7) is satisfied.

\section{Conclusions}

1. The two types of filters considered with the parameters calculated by the classical and neural network scheme do not have an ideal frequency response.

2. With the widening of the intermediate band between the boundary frequency of pass band and the boundary frequency of attenuation band of the filter, the non-uniformity of the frequency response in the pass band decreases.

3. The neural network method of approximation of the filter's transfer function is not inferior in accuracy to the classical method and has a significant simplicity especially in the implementation of recursive filters that do not require the use of transfer functions from the list of analog prototype filters (AF prototypes).

4. A neural network software product has been developed for calculating the parameters of a digital filter according to the specified requirements for filtering band boundaries, applicable to two types of digital filters.

\section{References}

1. A VLSI Architecture for Image Registration in Real Time (Based on systolic array) 15, (2007) http://ieeexplore.ieee.org/ iel5/92/4292150/04292156.pdf

2. FSBMA using FPGA for Real Time Applications, IJCSNS International Journal of Computer, Science and Network Security 8(3), 4651 (2008)

3. G. Hegde, FPGA European Journal of Scientific Research 33(4), 606-616 (2009) ISSN $1450-216 \mathrm{X}$

4. K. Samuel, M. Moore, Adding cores slows data-intensive applications, http://spectrum.ieee.org (2008)

5. V. Kankhva, MATEC Web of Conferences 106, 08027 (2017) doi: $10.1051 /$ matecconf/201710608027

6. I.V. Ilin, A. Lepekhin, A.I. Levina, O.Yu. Iliashenko, Advances in Intelligent Systems and Computing 692, 1306-1314 (2018) doi: 10.1007/978-3-319-70987-1_138

7. A.K. Orlov, I.Y. Chubarkina, MATEC Web of Conferences 106, 08015 (2017) doi: 10.1051/matecconf/201710608015 
8. D. Silka, V. Kankhva, MATEC Web of Conferences 106, 08031 (2017) doi: 10.1051/matecconf/201710608031

9. I.G. Lukmanova, M.Y. Mishlanova, International Journal of Economics and Financial Issues 5, 208-216 (2015)

10. G. Taveggia, A. Borboni, C. Mulé, J.H. Villafañe, S. Negrini, International Journal of Rehabilitation Research 39(1), 29-35 (2016) doi: 10.1097/MRR.0000000000000137 